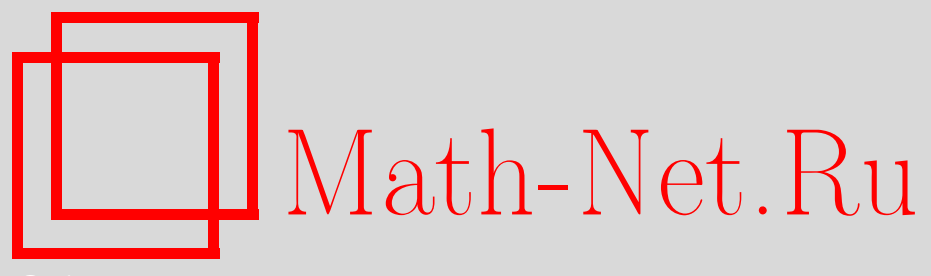

А. В. Пургин, Дистрибутивные и геометрические решетки правых делителей линейных обыкновенных дифференциальных операторов, УМН, 2009, том 64, выпуск $3,185-186$

DOI: https://doi.org/10.4213/rm9287

Использование Общероссийского математического портала Math-Net.Ru подразумевает, что вы прочитали и согласны с пользовательским соглашением http: //www . mathnet.ru/rus/agreement

Параметры загрузки:

IP : 54.157 .27 .8

26 апреля 2023 г., 13:49:28

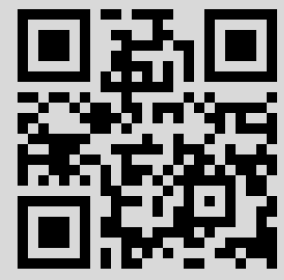




\section{Дистрибутивные и геометрические решетки правых делителей линейных обыкновенных дифференциальных операторов}

\section{А. В. Пургин}

В этой заметке мы рассматриваем некоторые алгебраические вопросы теории факторизации линейных обыкновенных дифференциальных операторов (ЛОДО) с коэффициентами из поля рациональных функций $Q(x)$, [1]-[4]. Известно [3], что произвольный ЛОДО разлагается на неприводимые множители с коэффициентами в том же поле $Q(x)$ с конечным числом параметров. Относительно недавно был получен алгоритм [3], который перечисляет все возможные факторизации заданного ЛОДО с рациональными коэффициентами.

В данной заметке для исследования структуры всех возможных правых делителей ЛОДО используются методы теории частично упорядоченных множеств. Адекватным представлением структуры данных, выдаваемых упомянутым выше алгоритмом нахождения всех факторизаций данного оператора, является решетка - частично упорядоченное множество, в котором для любых двух элементов существует их точная верхняя и точная нижняя грань [5].

Символом $Р$ будем обозначать ЛОДО

$$
P=D^{n}+f_{1}(x) D^{n-1}+\cdots+f_{n}(x), \quad D=\frac{d}{d x},
$$

где $f_{s}(x)$ принадлежат полю $Q(x)$.

Введем отношение частичного порядка на множестве всех правых делителей произвольного ЛОДО $P$ следующим образом: пусть $P_{1}$ и $P_{2}-$ некоторые правые делители оператора $P$, будем говорить, что $P_{1} \leqslant P_{2}$, если оператор $P_{1}$ является правым делителем оператора $P_{2}$. Частично упорядоченное множество правых делителей произвольного ЛОДО $P$ является решеткой с операциями взятия точной нижней грани $\inf \left\{P_{1}, P_{2}\right\}=\operatorname{rGCD}\left(P_{1}, P_{2}\right)$ и точной верхней грани $\sup \left\{P_{1}, P_{2}\right\}=\operatorname{rLCM}\left(P_{1}, P_{2}\right)$, где $\operatorname{rGCD}\left(P_{1}, P_{2}\right)$ обозначает оператор, являющийся правым наибольшим общим делителем ненулевых операторов $P_{1}$ и $P_{2}$, a $\operatorname{rLCM}\left(P_{1}, P_{2}\right)$ - оператор, являющийся их правым наименьшим общим кратным [4].

Пусть $P=P_{1} \circ P_{2} \circ \cdots \circ P_{i} \circ P_{i+1} \circ P_{i+2} \circ \cdots \circ P_{k}-$ некоторая факторизация ЛОДО $P$ на неприводимые множители $P_{i}$. Для любого $i \in\{1, \ldots, k\}$ обозначим символом $\left\langle P_{i}\right)$ следующий оператор: $\left\langle P_{i}\right)=P_{i} \circ P_{i+1} \circ P_{i+2} \circ \cdots \circ P_{k}-$ именно он и является элементом решетки правых делителей оператора.

ОПредЕлЕниЕ 1. Оператор $P$ называется даламберовым, если все его неприводимые множители $P_{i}$ являются операторами первого порядка: $P_{i}=D+a_{i}(x)$, где $a_{i}$ принадлежат дифференциальному полю рациональных функций.

ОПРеДЕЛЕниЕ 2 [3]. Два (для простоты неразложимых) оператора $P$ и $Q$ будем называть перестановочными в произведении $P \circ Q$, если $P \circ Q=Q_{1} \circ P_{1}, Q_{1} \neq P$, $P_{1} \neq Q$.

Будем говорить, что у ЛОДО $P$ все факторы перестановочны, если в любой факторизации оператора $P$ для любых двух соседних правых делителей $\left\langle P_{i}\right)$ и $\left\langle P_{i+1}\right)$ операторы $P_{i}$ и $P_{i+1}$ перестановочны.

Необходимые стандартные определения таких понятий теории решеток, как "дистрибутивность", "геометрическая решетка", "модулярная решетка", "отношение покрытия", которые существенны для изучения решеток правых делителей ЛОДО, можно посмотреть в [5].

Как легко показать, решетка правых делителей любого ЛОДО модулярна. 
Символом $M_{3}$ обозначим решетку, изоморфную 5 -элементной модулярной решетке, состоящей из наименьшего элемента $\widehat{0}$, наибольшего элемента $\widehat{1}$ и трех элементов $a, b, c$ таких, что $a, b, c$ покрывают элемент $\widehat{0}$ и покрываются элементом $\widehat{1}$.

Следующая лемма представляет самостоятельный интерес в теории решеток.

Лемма 1. Пусть $L$ - модулярная решетка конечной высоты. Тогда если решетка $M_{3}$ вложима в решетку $L$, то $M_{3}$ вложима в некоторый интервал высоты 2.

Получены следующие результаты.

Теорема 1. Для ЛОДО Р следующие условия эквивалентны: 1) решетка правых делителей ЛОДО Р дистрибутивна; 2) все факторы оператора Р непараметризованы.

Теорема 2. Пусть L - произвољная конечная дистрибутивная решетка высоты n. Тогда существует даламберов ЛОДО Р порлдка $n$ (не обязательно единственный) с коэффициентами из дифференциального поля рачиональных функиий $Q(x)$ такой, что решетка $L_{P}$ его правых делителей изоморфна $L$.

Теорема 3. Решетка правых делителей ЛОДО Р геометрическая тогда и только тогда, когда у оператора $P=P_{1} \circ \cdots \circ P_{n}$ все факторы перестановочны.

\section{Список литературы}

[1] M. V. Hoeij, J. Symbolic Comput., 24:5 (1997), 537-561. [2] O. Ore, Ann. of Math. (2), 34:3 (1933), 480-508. [3] S. P. Tsarev, Proceedings of ISSAC (Zürich, 1996), ACM Press, New York, 1996, 226-231. [4] С. А. Абрамов, С. П. Царев, Программирование, 23:1 (1997), 59-67; англ. пер.: S. A. Abramov, S. P. Tsarev, Program. Comput. Software, 23:1 (1997), 45-50. [5] Г. Гретцер, Общая теория решеток, Мир, М., 1982; пер. с англ.: G. Grätzer, General lattice theory, Pure Appl. Math., 75, Academic Press, New York-London, 1978.

\section{А. В. Пургин (А. V. Purgin)}

Красноярский государственный педагогический университет им. В. П. Астафьева

E-mail: pav1972@yandex.ru
Представлено В. М. Бухштабером Принято редколлегией 07.04.2009 